\title{
"Análisis de la motivación en los trabajadores y su repercusión en el nivel de satisfacción de los clientes en el Hotel Casa Suayay - Miraflores 2017"
}

\section{"Analysis of the motivation in the workers and their repercussion in the level of satisfaction of customers at the Casa Suayay Hotel - Miraflores 2017"}

\author{
CPC. Delia Elsa Cueva Julca / David Rodrigo, Muñoz Apari \\ Facultad de Ciencias Empresariales / Universidad Alas Peruanas \\ Correo-e: d_cueva1@ @otmail.com
}

\section{RESUMEN}

Objetivo: La presente investigación tiene como objetivo analizar el nivel de motivación de los trabajadores del Hotel Casa Suyay y su repercusión en la satisfacción, realizando encuestas para poder medir el grado de motivación de los trabajadores y desarrollar medidas correctivas para aliviar los problemas que se dan dentro del hotel. Materiales y Métodos: La investigación es aplicada de forma descriptiva con un diseño no-experimental porque solo se va a observar y tomar datos de las encuestas, se trabajó con una muestra de 15 trabajadores y 100 huéspedes. La técnica a emplearse en la presente investigación es de encuesta y el instrumento cuestionario. Resultados: EJECUCIÓN Y DESARROLLO: El trabajo de recolección de datos duró 5 días, el $65 \%$ del personal evaluado se encuentran motivados dentro del hotel y el 35\% no tanto. EFECTOS: el 56\% de los trabajadores encuestados, tienen la necesidad de que haya un cambio dentro de la empresa para que puedan realizar su trabajo con una mayor motivación y por ende con una mayor eficacia. Conclusiones: Los trabajadores del Hotel Casa Suyay creen que pueden mejorar su desempeño cuando reciban un mayor impulso por parte de la empresa.

Palabras Claves: Motivación, satisfacción.

\begin{abstract}
Objective: The objective of this research is to analyze the level of motivation of the workers of Hotel Casa Suyay and its repercussion on satisfaction, carrying out surveys to be able to measure the motivation of the workers and to develop corrective measures to alleviate the problems that occur inside the hotel. Materials and Methods: The research is applied in a descriptive way with a nonexperimental design because only the data will be taken and taken from the surveys, a sample of 15 workers and 100 guests was worked. Results: IMPLEMENTATION AND DEVELOPMENT: Data collection work lasted 5 days, 65\% of the staff evaluated were motivated within the hotel and 35\% were not so much. EFFECTS: $56 \%$ of the workers surveyed have a need for a changé within the company so that they can carry out their work with greater motivation and therefore with greater efficiency. Conclusions: The workers at Hotel Casa Suyay believe that they can improve their performance when they receive a greater impulse from the company.
\end{abstract}

Keywords: Motivation, satisfaction. 


\section{Introducción}

En los hoteles y en otros tipos de negocios es muy importante el factor humano para poder alcanzar las metas y objetivos trazados, y si se quiere que esto se logre los trabajadores tienen que estar motivados para cumplir un buen desempeño y poder satisfacer a los clientes, ya que una buena motivación se ve reflejado en una buena satisfacción por parte de los clientes, y el resultado que se obtiene es de vital importancia para la empresa.

La motivación es muy importante dentro de una empresa, porque permite que los objetivos trazados, en la misión y visión puedan cumplirse, así también es muy importante el clima laboral en donde se trabaja, la relación entre compañeros de trabajo, la interrelación y el feedback entre la cabeza de la empresa y la parte operativa de la misma, manejar un personal que está completamente motivado se identifica con la empresa, esto se refleja en la interacción con los huéspedes, la satisfacción de los mismos es muy importante ya que permite a corto y largo plazo cumplir con los objetivos y metas.

La motivación es parte fundamental para ejecutar cualquier actividad ya que es la que permite que una persona actúe y se comporte de una manera determinada, en términos laborales, podemos decir que la motivación es el motor que impulsa a que el colaborador realice bien o mal las tareas.

Dentro de las compañías, independientemente del tamaño de la misma, se daba más valor al producto o servicio que se comercializaba y en segundo plano quedaba el cliente interno, es decir los colaboradores, se restaba valor a lo que el talento humano necesitaba para poder desempeñar eficientemente las funciones y como se sentía al ejercer el cargo asignado para llegar a ser eficaz.

Las empresas se preocupaban más en realizar estudios de mercados, conocer las nuevas necesidades de las personas y de esta manera desarrollar un producto o servicio para comercializarlo, pero no se realizaba un estudio en el que se conozca si el colaborador estaba suficientemente motivado para el trabajo.

Sabemos que la clave del éxito para una empresa es tener clientes fieles y satisfechos, esto solo se lo logra brindándoles un buen servicio a través de los colaboradores, por lo tanto, es importante que el talento humano actúe de manera positiva ante cualquier tarea encomendada.

Para tener un personal motivado es primordial asignar recursos tangibles, intangibles y humanos, ver esta asignación como una inversión y no como un gasto como generalmente lo ven los directores o jefes. Al tener un personal motivado este será más eficiente al momento de ejecutar las tareas, lo cual traerá consigo mayor rédito a la empresa tanto económicamente como estructuralmente. Brindar un buen servicio se lleva a cabo por medio de capacitaciones, incentivos, un buen ambiente laboral, esto genera que el colaborador trabaje motivado y produzca más. Cabe recordar que es indispensable aplicar KPI, apropiado.

Para optimizar esto es necesario saber que evaluaciones de desempeño y motivación con equidad debe de hacerse.

La motivación es todo un arte, el saber mostrar el aprecio reciproco y brindar la recompensa apropiada al equipo de trabajo es lo pertinente; pero recordemos que cada incentivo debe ser dirigido para sujetos pensantes diferentes y que en este mundo solo hay cuatro cosas que mueven este mundo y son amor, ciencia, Fe y dinero.

Reconocer que desea nuestro colaborador ese punto neurálgico de análisis y de precisión.

Palabras claves: Rédito, Incentivo, Motivación, KPI, Eficiente, Eficaz.

La presente investigación tiene una relevancia social ya que permite conocer la motivación de los trabajadores y la satisfacción de los huéspedes, supliendo estas mismas. Logrando de esta manera un mejor desempeño del trabajador y la satisfacción de los huéspedes.

Tiene relevancia académica ya que contribuirá a la escuela académico profesional de Turismo, Hotelería y Gastronomía de la Universidad Alas Peruanas, dado que esta investigación aportara conocimiento académico al alumnado de esta facultad.

\section{Materiales y métodos}

La presente investigación es de tipo observable y descriptiva, porque la investigación pretende analizar la motivación en los trabajadores y la satisfacción de los huéspedes.

Es la estrategia para obtener la información deseada. El diseño de esta investigación es No experimental por que no manipulo la variable, solo se realiza recaudación de datos e información para la investigación.

SAMPIERI, FERNANDEZ Y BAPTISTA (2010) afirma que el diseño de investigación no experimental es "la investigación que se realiza sin manipular deliberadamente variables" (p.149) 
Lo que se hace en la investigación no experimental es observar fenómenos tal como se dan en su contexto natural, para posteriormente analizarlos. Se clasifica

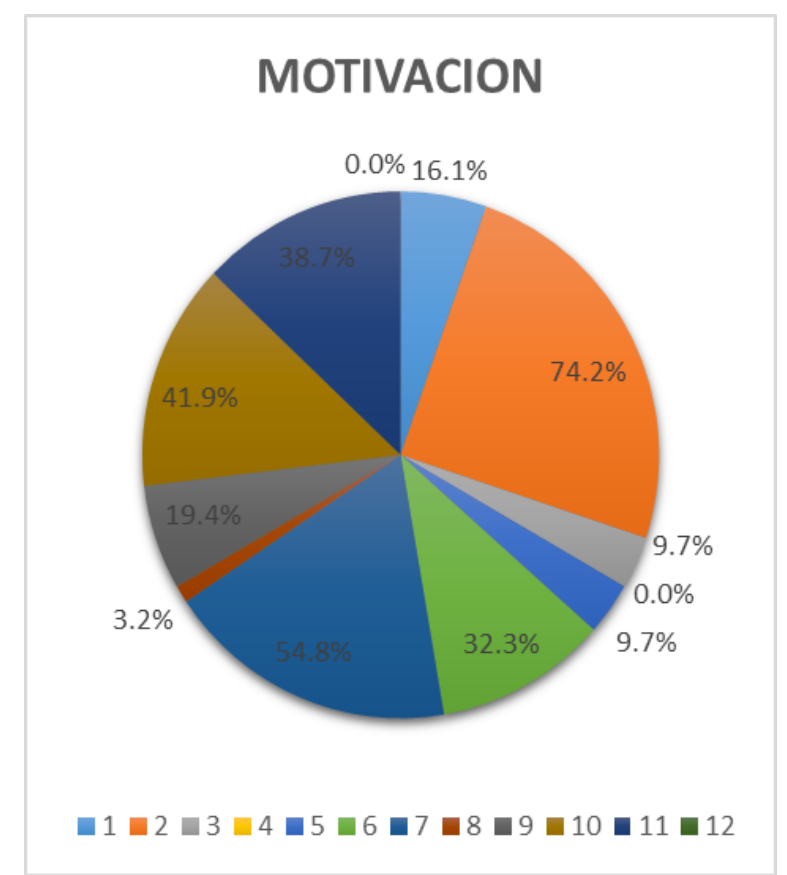

por su dimensión temporal o el número de momentos o puntos en el tiempo, en los cuales se recolectan datos: transaccionales y longitudinales.

\section{Gráfica $N^{\circ}$ 1: Motivación/Factores económicos Elaboración propia.}

Análisis. La presente tabla permite ver que los trabajadores del Hotel Casa Suyay se sienten satisfechos con

Respecto al salario, un $67.7 \%$, sin embargo existe un $16.1 \%$ de trabajadores insatisfechos, esto puede ser porque aún no están en planilla y trabajan bajo recibo por honorarios.

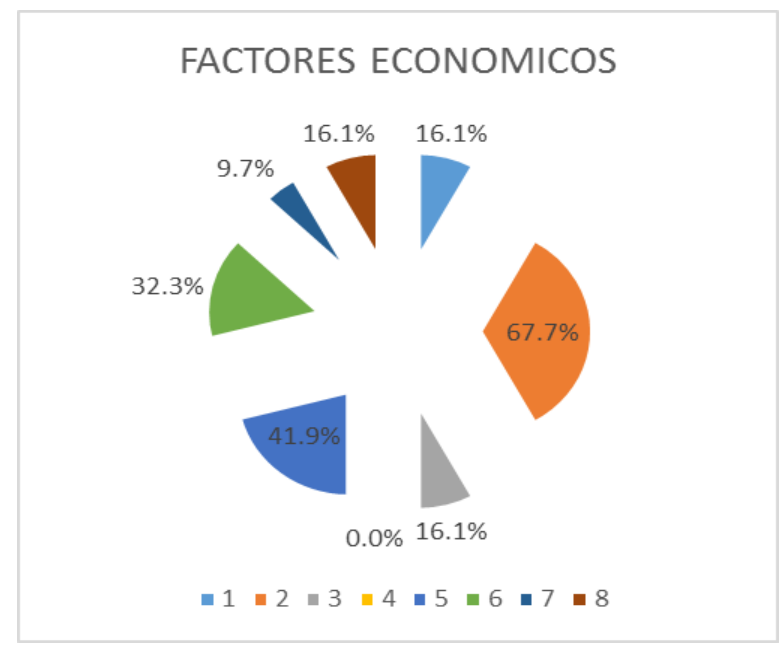

Gráfica $N^{\circ}$ 2: Motivación Elaboración propia.
Análisis. La presente tabla permite ver que los trabajadores del Hotel Casa Suyay cuentan con un $74.2 \%$ de satisfacción de acuerdo a la motivación para realizar su trabajo, además de que un $41.9 \%$ se sienten a gusto en el área que operan, sin embargo, un $54.8 \%$ siente que su trabajo no es reconocido por sus superiores lo que puede desmotivarlos.

\section{MOTIVACION EXTRINSECA}

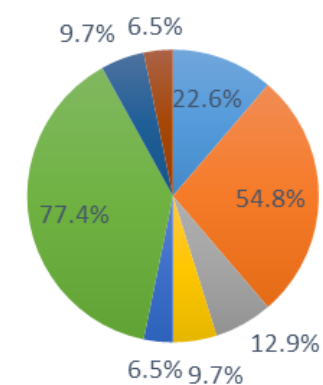

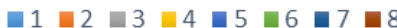

Gráfico $\mathrm{N}^{\circ}$ 3: Conocimiento - Post test Porcentaje. Elaboración propia.

Análisis. La presente tabla permite ver que los trabajadores del Hotel Casa Suyay cuentan con un $54.8 \%$ de satisfacción de acuerdo al horario de trabajo, además

$77.4 \%$ sienten que trabajan en condiciones óptimas, esto nos da a entender que el hotel se preocupa por el área de trabajo de sus trabajadores

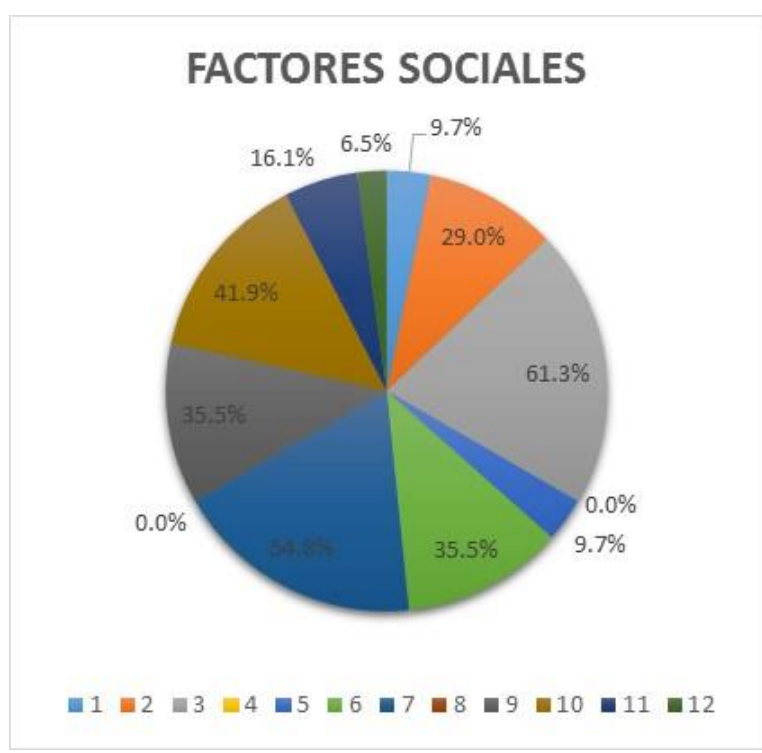

Gráfico $N^{\circ}$ 4: Factores sociales - Post test porcentajes.

Análisis. La presente tabla permite ver que un gran porcentaje de los trabajadores del Hotel Casa Suyay se sienten insatisfechos y tienen miedo al poder expresar sus ideas para mejora del trabajo, 61.3\%. Además podemos observar que la comunicación dentro de la empresa es baja, con un $54.8 \%$ de 
insatisfacción, sin embargo con respecto al clima laboral un $41.9 \%$ de los trabajadores se sienten satisfechos, esto quiere decir que la empresa tiene bastante por trabajar en cuanto a la comunicación con sus trabajadores.

\section{Discusión}

Uno de los grandes problemas que poseen las empresas hoteleras actualmente es que el personal no se encuentra motivado, las empresas se centran en obtener rentabilidad económica y se olvidan del factor más importante por el cual estas se encuentra en funcionamiento, es decir, el trabajador; actualmente existe una corriente en varias empresas la cual consiste en tener un buen ambiente laboral, para así poder tener satisfecho a los empleados, y estos sientan que sus aportes son importantes para la empresa.

Los resultados de nuestra investigación demuestran que en cuanto a la motivación del personal que se encuentran laborando en el hotel se puede decir que los mencionados trabajadores se encuentran satisfechos, es decir, se encuentran motivados, ya que la empresa en la que laboran, toman en cuenta sus aportes, se reconocen las tareas que realizan, y se sienten satisfecho con las labores que desarrollan, de igual manera con las vacaciones y el horario de trabajo, pero existe un grupo de trabajadores que no están satisfechos con las condiciones de trabajo como son el horario y las vacaciones, probablemente esto ocurre porque poseen horarios altamente rotativos, y además de no cumplir las ocho horas de trabajo que se encuentran reglamentadas por ley.

Los trabajadores mencionaron que cuando ingresa un nuevo trabajador a laborar en dicha empresa hotelera se sienten satisfechos al ayudarlos, la misma situación se presenta cuando un compañero de trabajo recibe un reconocimiento por alguna labor realizada, podemos llegar a la conclusión de que el personal que labora en el hotel posee un ambiente ameno dentro de la empresa, es decir, por más que cada uno de los trabajadores tenga su tarea definida, el trabajo en conjunto que se da es vital para el desarrollo de la empresa en la que se encuentran laborando; hay que recalcar que existe un grupo de trabajadores que están insatisfecho con la situación anteriormente mencionada lo cual generaría un ambiente hostil para poder laborar, esta situación ocurriría probablemente porque este personal no tiene buena relación laboral con sus compañeros de trabajo y puede considerar que estos son su competencia .

La motivación es el proceso que impulsa a una persona a actuar de una determinada manera o por lo menos origina una propensión hacia un comportamiento específico, los trabajadores del Hotel Casa Suyay indicaron que se encuentran motivados laborando para la mencionada empresa, esta situación genera que el personal se sienta comprometido con los objetivos de la empresa, lo cual se traducirá en empleados comprometidos y con ganas de sumar.

En cuanto a la promoción en el trabajo, la mayoría de los empleados indicaron que sienten un nivel de insatisfacción con la posibilidad de ascenso laboral y con la oportunidad de desarrollarse laboralmente dentro de la mencionada empresa hotelera, esta situación ocurre porque posiblemente, los empleados posean un contrato por un corto periodo de tiempo, lo cual no les permite tener las posibilidades de desarrollarse profesionalmente dentro de la empresa, pero si el personal permanece laborando en el hotel por varios años, es posible que logren tener un ascenso laboral y de este modo se desarrollaran laboralmente dentro de la empresa.

\section{Conclusiones}

El personal que labora en el hotel se encuentra motivado al momento de realizar sus funciones dentro de cada área, así como también se encuentran satisfechos laborando para dicha empresa, esto se ve reflejado en el servicio ofrecido por algunas áreas del hotel.

Al encontrarse el personal motivado esto le permite incrementar su desempeño y rendimiento logrando desarrollar su trabajo de forma eficiente, lo que genera que se ofrezca un buen servicio siempre buscando el bienestar de los huéspedes repercutiendo de manera positiva en el nivel de satisfacción en los clientes, de tal forma que el servicio recibido supere sus expectativas.

La investigación nos ayudó a determinar los factores presente en cuanto al servicio ofrecido al huésped, que influyeron de manera positiva como el servicio ofrecido por los trabajadores, quienes demuestran predisposición para ayudar a estos, así como también el interés para solucionar sus inquietudes, esta situación genera que el personal se encuentre pendiente.

\section{Recomendaciones}

La empresa debe de establecer un sistema de motivación e incentivos que cubran las expectativas de los miembros de la empresa, se deben emplear estrategias para la unión de los empleados, estrategias motivacionales para que los empleados se involucren aún más, también crear hábitos emprendedores y de comunicación con los superiores para que el personal sienta mayor libertad a la hora de opinar y expresar sus ideas.

La gerencia del Hotel Casa Suyay debe enfocarse en realizar un programa de capacitación para los empleados contribuyendo al desarrollo de estos tanto en el ámbito personal como profesional, así los 
trabajadores puedan adquirir conocimientos y sientas una mayor motivación a la hora de realizar su trabajo.

El hotel debe de establecer jefatura en cada área, delegar funciones y responsabilidades, para que así se puedan realizar reuniones y verificar el desempeño laboral de cada uno de los trabajadores.

\section{Referencias bibliográficas}

[1] Chiavenato Idalberto. (2007). Administración de recursos humanos. (5ta $\mathrm{Ed})$.

[2] Kotler, Philip. Dirección de Mercadotecnia. Edición 8va, Págs. 40, 41.

[3] Kotler, Philip y Armstrong. Fundamentos de Marketing, Edición 6ta, Págs. 10, 11.

[4] Mcgregor, Douglas. (1971) El Lado Humano de las Organizaciones. Ed. Mac Graw Hill. Colombia.

[5] Sandoval, Washington. (2007). La motivación. Universidad Politécnica del Ejército. Ecuador.

[6] William B. Werther, Jr. Keith Davis. (1996). Administración de Personal y Recursos Humanos (5ta Ed.) Ed. Mac Graw - Hill. México

[7] Palomino, Teodosio. (1995). Administración de Recursos Humanos. Editorial Juris Laboral. Lima - Perú. Pág. 59. 